\title{
The 1-vertex transfer matrix and accurate estimation of channel capacity
}

\author{
S. Friedland, P.H. Lundow and K. Markström
}

\begin{abstract}
The notion of a 1-vertex transfer matrix for multidimensional codes. It is shown that the capacity of such codes, or the topological entropy, can be expressed as the limit of the logarithm of spectral radii of 1 -vertex transfer matrices. Storage and computations using the 1-vertex transfer matrix are much smaller than storage and computations needed for the standard transfer matrix.

The method is applied to estimate the first 15 digits of the entropy of the 2-dimensional $(0,1)$ run length limited channel.

In order to compare the computational cost of the new method with the standard transfer matrix and have rigorous bounds to compare the estimates with a large scale computation of eigenvalues for the $(0,1)$ run length limited channel in 2 and 3 dimensions have been carried out. This in turn leads to improvements on the best previous lower and upper bounds for that channel.
\end{abstract}

2000 Mathematics Subject 2000 Mathematics Subject Classification: 05A16, 28D20, 37M25, 82B20

Keywords and phrases: Channel capacity, transfer matrices, multi-dimensional codes, optical storage.

\section{INTRODUCTION}

In classical information theory the emphasis was on onedimensional channels. This is the natural channel type when one is considering sequential information transfer and information storage on effectively one-dimensional media, such as a magnetic tape. However, in modern information technology higher dimensional channels are becoming more and more important. The simplest example is of course information storage on a surface, as on a CD or DVD. Future devices seem likely to move on to three-dimensional storage media as well.

Just as for the classical one-dimensional channels one still wishes to construct codes to protect data from errors and to find the channel capacity given by the code. In higher dimensions computing the exact capacity becomes much more difficult and even for very simple codes we do not know the exact value. The typical example is the run-length limited channel. Consider a two-dimensional square grid where each point can be assigned a value of either 0 or 1 with the restriction that two points which differ by one in exactly one coordinate cannot both be 1 . This channel has been studied in both information theory [NZ99], combinatorics [CW98] and physics [BET80]. In statistical physics this has been studied in

Department of Mathematics, Statistics and Computer Science, University of Illinois at Chicago, Chicago, Illinois 60607-7045, USA, < friedlan@uic.edu >

Department of Theoretical Physics, AlbaNova University Center, KTH, SE10691 Stockholm, Sweden, <phl@kth.se>.

Department of Mathematics and Mathematical Statistics, UmeåUniversity, SE-901 87 Umeå, Sweden, <Klas.Markstrom@math.umu.se> . terms of lattices gases, primarily through non-rigorous methods and simulation. However a sequence of authors [CW98], [KZ99], [FP05], [NZ99], [NZ03] have developed methods for establishing rigorous upper and lower bounds by using transfer matrices. The drawback with these methods is that the cost of achieving a given precision is exponential in terms of the precision. It is possible to use symmetries [LM08] to speed up the computation but typically the cost remains exponential, with improved constants.

Hence it would be desirable to be able to establish good estimates for the capacity of a code before deciding whether to invest the time needed to bound its capacity rigorously. In this paper we present a method for obtaining such estimates. The method uses a so called 1-vertex transfer matrix which adds one vertex at a time to the graph, rather than whole rows as in the standard method. We prove that under certain conditions on the code the largest eigenvalue of this matrix converges to the channel capacity. The drawback is that we do not get easily computable bounds for the capacity, but as a practical example will demonstrate one can get estimates which agrees with the rigorous bounds at a fraction of the computational cost. Hence this method can be used as a tool for quickly testing many different candidate codes in order to identify promising coding schemes, for which one can later establish rigorous bounds as well.

In order to help evaluate the accuracy our new method we have also improved the known bounds for the capacity of the two and three-dimensional run-length limited channels, or hard core-lattice gases as they are known in the physics literature.

\section{A. Mathematical preliminaries}

Many channels and models from statistical physics can be described in terms of restricted colourings of an underlying graph, often a lattice such as $\mathbb{Z}^{d}$, or more generally in terms of weighted graph homomorphisms $\left[\mathrm{BCL}^{+} 06\right]$, [FLS07], [LM08].

We now explain the main ideas and results of our paper for the two-dimensional grid $\mathbb{Z}^{2}$, where the colouring conditions are symmetric and isotropic. Let $\Delta=(V, E), V=\{1, \ldots, k\}$ be an undirected graph on $k$ vertices, where vertex $i$ is identified with the colour $i$. Two neighbouring points $\mathbf{p}=$ $\left(p_{1}, p_{2}\right)^{\top}, \mathbf{q}=\left(q_{1}, q_{2}\right)^{\top} \in \mathbb{Z}^{2}$, i.e. $\left|p_{1}-q_{1}\right|+\left|p_{2}-q_{2}\right|=1$, can be coloured in colours $i, j \in V$ if and only if $(i, j) \in E$. We assume that $\Delta$ has no isolated vertices.

The standard transfer matrix is defined as follows. For a positive integer $n \in \mathbb{N}$ denote by $\langle n\rangle=\{1, \ldots, n\}$. Then $\phi:\langle n\rangle \rightarrow V$ is viewed as a colouring of $\langle n\rangle$ in $k$ colours. View $\langle n\rangle$ as $n$ points on the real line, where $i, j \in\langle n\rangle$ are 
neighbours if and only if $|i-j|=1$. Then $\phi \in \mathrm{C}_{\Delta}(\langle\mathrm{n}\rangle)$ is an allowable colouring if $(\phi(i), \phi(j)) \in E$ whenever $|i-j|=1$. Let $N(n)=\# \mathrm{C}_{\Delta}(\langle\mathrm{n}\rangle)$ be the cardinality on $\mathrm{C}_{\Delta}(\langle\mathrm{n}\rangle)$. Note that $N(n)=O\left(\rho(\Delta)^{n}\right)$, where $\rho(\Delta)$ is the spectral radius of $\Delta$. Let $\phi, \psi \in \mathrm{C}_{\Delta}(\langle\mathrm{n}\rangle)$ be two allowable $\Delta$ colourings of $\langle n\rangle$. View $(\phi, \psi)$ as a colourings of integer strip $\tau:\langle n\rangle \times\langle 2\rangle \subset \mathbb{Z}^{2}$, where $\tau((i, 1))=\phi(i), \tau((i, 2))=\psi(i)$ for $i=1, \ldots, n$. Let $t_{\phi, \psi}=1$ if $\tau$ is a $\Delta$-allowed colouring, i.e. $(\phi(i), \psi(i)) \in$ $E$ for $i=1, \ldots, n$, and otherwise $t_{\phi, \psi}=0$. Then $T_{n, 2}=$ $\left[t_{\phi, \psi}\right]_{\phi, \psi \in \mathrm{C}_{\Delta}(\langle\mathrm{n}\rangle)} \in\{0,1\}^{N(n) \times N(n)}$ is the transfer matrix, which defines the allowable colourings of the infinite strip $\langle n\rangle \times \mathbb{Z} \subset \mathbb{Z}^{2}$. An important and useful observation is that $T_{n, 2}$ is a symmetric matrix.

Let $\rho\left(T_{n, 2}\right)$ be the spectral radius of $T_{n, 2}$. It is well known that the sequence $\log \rho\left(T_{n, 2}\right)$ is subadditive in $n$. Hence the following limit exists, and it is called the topological entropy of $\mathbb{Z}^{2}$ with respect to $\Delta$, e.g. [Fri97], [Fri03], [FP05]:

$$
h_{2}(\Delta):=\lim _{n \rightarrow \infty} \frac{\log \rho\left(T_{n, 2}\right)}{n} .
$$

Furthermore, one has the following known upper and lower bounds, see e.g. [Fri97], [Fri03]

$$
\begin{aligned}
\frac{\log \rho\left(T_{p+2 q+1,2}\right)-\log \rho\left(T_{2 q+1,2}\right)}{p} & \leq h_{2}(\Delta) \leq \frac{\log \rho\left(T_{n, 2}\right)}{n}, \\
& 1 \leq p, n, 0 \leq q . \quad \text { (I.2) }
\end{aligned}
$$

In many theoretical derivations, e.g. [Lie67], it is useful to consider the periodic colouring $\mathrm{C}_{\Delta \text {,per }}(\langle\mathrm{n}\rangle) \subset \mathrm{C}_{\Delta}(\langle\mathrm{n}\rangle)$, where each colouring $\phi \in \mathrm{C}_{\Delta}(\langle\mathrm{n}\rangle)$ satisfies the additional condition $(\phi(1), \phi(n)) \in E$. So $\mathrm{C}_{\Delta \text {,per }}(\langle\mathrm{n}\rangle)$ is a $\Delta$-allowable colouring of the torus $\mathbb{Z} /\langle n\rangle$. Let $N(n$, per $)=\# \mathrm{C}_{\Delta \text {,per }}(\langle\mathrm{n}\rangle)$ be the number of periodic $\Delta$-colouring of $\mathrm{C}_{\Delta \text {,per }}(\langle\mathrm{n}\rangle)$. Then $T_{n, 2, \text { per }}=\left[t_{\phi, \psi}\right]_{\phi, \psi \in \mathrm{C}_{\Delta, \text { per }}(\langle\mathrm{n}\rangle)} \in\{0,1\}^{N(n, \text { per }) \times N(n, \text { per })}$ is the transfer matrix for the allowable colourings of the infinite torus $(\mathbb{Z} /\langle n\rangle) \times \mathbb{Z}$ by $\Delta$. Note that $T_{n, 2 \text {,per is a }}$ principal submatrix of $T_{n, 2}$, hence symmetric. Furthermore $\rho\left(T_{n, 2, \text { per }}\right) \leq \rho\left(T_{n, 2}\right)$. Also, the following inequalities hold [FP05]

$$
\begin{aligned}
\frac{\log \rho\left(T_{p+2 q, 2, \mathrm{per}}\right)-\log \rho\left(T_{2 q, 2, \mathrm{per}}\right)}{p} & \leq h_{2}(\Delta) \leq \\
\frac{\log \rho\left(T_{2 n, 2, \mathrm{per}}\right)}{2 n}, 0 & \leq q, 1 \leq p, n,
\end{aligned}
$$

where $\rho\left(T_{0,2, \text { per }}\right)=\rho(\Delta)$. Note that the upper bound in (I.3) is an improvement of the upper bound in (I.2) for an even $n$.

We now define the 1-vertex transfer matrix

$$
S_{n, 2}=\left[s_{\phi, \psi}\right]_{\phi, \psi \in \mathrm{C}_{\Delta}(\langle\mathrm{n}\rangle)} \in\{0,1\}^{N(n) \times N(n)},
$$

where

$$
\begin{array}{r}
s_{\phi, \psi}=1 \text { iff } \psi(i)=\phi(i+1) \text { for } i=1, \ldots, n-1, \text { and } \\
(\phi(1), \psi(n)) \in E .
\end{array}
$$

$S_{n, 2}$ is the transfer matrix corresponding to the $\Delta$ colouring of $\mathbb{Z}$ winded up on a slanted torus, given by $\langle n+1\rangle \times \mathbb{Z}$, where the points $(n+1, i)$ and $(1, i+1)$ are identified for each $i \in \mathbb{Z}$. Note that $S_{n, 2}$ is a sparse matrix, where each row and column of $S_{n, 2}$ has at most $k$ neighbours. We show that

$$
\lim _{n \rightarrow \infty} \log \rho\left(S_{2 n+1,2}\right)=h_{2}(\Delta) .
$$

Thus $\log \rho\left(S_{n, 2}\right)$ is an approximation of $h_{2}(\Delta)$, and it can be computed much faster, and with much less memory than $\log \rho\left(T_{n, 2}\right)$ and $\log \rho\left(T_{n, 2, \text { per }}\right)$. The only drawback for computing the values of $\log \rho\left(S_{n, 2}\right)$, is that we do not have very sharp inequalities of the type (I.2) or (I.3). One of the reasons is that $S_{n, 2}$ is not a symmetric matrix and can have nonreal eigenvalues. We do have the following inequalities

$$
\begin{aligned}
& \frac{\log \rho\left(T_{n-1,2, \text { per })}\right)}{n} \leq \log \rho\left(S_{n, 2}\right) \leq \\
& \min \left(\frac{\log \rho\left(T_{n, 2}\right)}{n}, \frac{\log \rho\left(T_{n+1,2, \text { per }}\right)}{n}\right), 2 \leq n,
\end{aligned}
$$

which imply (I.6).

The undirected graph $\Phi$, which can be viewed as a symmetric digraph on two vertices

$$
\Phi=(V, E), \quad V=\{1,2\}, E=\{(1,2),(2,1),(2,2)\},
$$

corresponds to the checkerboard constraint used in [NZ03] and hard core lattice gas model in statistical mechanics. (Usually 2 is replaced by 0 .) We will use the 1 -vertex transfer matrix to give a heuristic estimate of the entropy of the two dimensional hard core $h_{2}(\Phi)$ up to 15 digits. Using the upper and lower bounds (I.2) and (I.3) we show that the 15 digits estimates agree with the correct values of the $h_{2}(\Phi)$. Previously the value of $h_{2}(\Phi)$ was known to 10 digits [NZ99].

\section{B. Overview}

We now briefly survey the contents of the paper. In $\S 2$ we give the full details of the two-dimensional isotropic symmetric case discussed above. In $\S 3$ we discuss the computational results for the two-dimensional checkerboard constraint/hard core lattice gas. In $\S 4$ we discuss the characteristic polynomial and the eigenvalues of $S_{n, 2}$ for the hard core model. In $\S 5$ we discuss a two-dimensional 1-vertex transfer matrix $P_{n, 2}$ for nonisotropic and nonsymmetric colouring of $\mathbb{Z}^{2}$. We show that $\log \rho\left(P_{n, 2}\right)$ is an estimate of the two dimensional-capacity, or entropy, if the colouring of $\mathbb{Z}^{2}$ has a friendly colour. In $\S 6$ we show that the results for two dimensions apply also to the three-dimensional 1-vertex matrix. In the last section we show that 1-vertex model can also be used to estimate the monomer-dimer model in any dimension $d$.

\section{THE TWO-DIMENSIONAL ISOTROPIC SYMMETRIC CASE}

Recall that the graph $\Delta=(V, E)$, where the set of vertices $V$ is identified with $k$ colours $\langle k\rangle$. We allow loops $(i, i) \in E$, which means that two adjacent vertices of $\mathbb{Z}^{2}$ can be coloured with the same colour $i$. If $i \in V$ is an isolated vertex, this means that no vertex in $\mathbb{Z}^{2}$ can be coloured in the colour $i$. Hence we will deal only with graphs $\Delta$ which do not have isolated vertices.

Theorem 2.1: Let $\Delta=(V, E)$ be an undirected graph on $\# V=k \geq 2$ vertices, where self-loops are allowed, with no isolated vertices. Then (I.7) and (I.6) hold. 
Proof: Identify the entry $s_{\phi, \psi}=1$ of $S_{n, 2}$ with an allowable $\Delta$-colouring of the following $L$ shape in $\mathbb{Z}^{2}$

$$
\begin{aligned}
& \omega:\{(1,1), \ldots,(n, 1),(1,2)\} \rightarrow\langle k\rangle, \\
& \omega((i, 1))=\phi(i), i=1, \ldots, n, \omega((1,2))=\psi(n) .
\end{aligned}
$$

More generally, consider the entries of the matrix $S_{n, 2}^{m}=$ $\left[s_{\phi, \psi}^{(m)}\right]_{\phi, \psi \in \mathrm{C}_{\Delta}(\langle\mathrm{n}\rangle)}$. Recall that

$$
s_{\phi_{1}, \phi_{m+1}}^{(m)}=\sum_{\phi_{1}, \ldots, \phi_{m+1} \in \mathrm{C}_{\Delta}(\langle\mathrm{n}+1\rangle)} \prod_{i=1}^{m} s_{\phi_{i}, \phi_{i+1}} .
$$

Clearly, $\prod_{i=1}^{m} s_{\phi_{i}, \phi_{i+1}}=0,1$. Then $\prod_{i=1}^{m} s_{\phi_{i}, \phi_{i+1}}=1$ if and only if one has a $\Delta$-allowable colouring $\tau \in \mathrm{C}_{\Delta}(\langle\mathrm{n}+\mathrm{m}\rangle)$ such that

$$
(\tau(i), \tau(j)) \in E \text { if }|i-j|=n .
$$

Assume that $\prod_{i=1}^{m} s_{\phi_{i}, \phi_{i+1}}=1$. Then we can colour the following part of $\langle n\rangle \times \mathbb{Z}$ :

$$
\begin{array}{r}
(1,1), \ldots,(n, 1),(1,2), \ldots,(1, q), \ldots,(l, q), \\
q=\left\lceil\frac{n+m}{n}\right\rceil, l=n+m-(q-1) n,
\end{array}
$$

using $\tau$. Let $\mathbf{1}=(1, \ldots, 1)^{\top}$ be a vector whose all coordinates are 1 . The number of coordinates of 1 will depend on the square matrix $A=\left[a_{i j}\right] \in \mathbb{R}^{M \times M}$. Observe that $\mathbf{1}^{\top} A \mathbf{1}=\sum_{i, j=1}^{M} a_{i j}$. Hence $\mathbf{1}^{\top} S_{n, 2}^{m} \mathbf{1}$ is the number of all $\Delta$ allowable colourings $\tau \in \mathrm{C}_{\Delta}(\langle\mathrm{n}+\mathrm{m}\rangle)$ satisfying the condition (II.2). In particular, $\mathbf{1}^{\top} S_{n, 2}^{(q-1) n} \mathbf{1}$ is the number of different colourings of the rectangle $\langle n\rangle \times\langle q\rangle$ induced by $\tau \in \mathrm{C}_{\Delta}$ (qn) satisfying (II.2). Similarly, $\mathbf{1}^{\top} T_{n, 2}^{q-1} \mathbf{1}$ is the number of $\Delta$ allowable colourings of the strip $\langle n\rangle \times q$. Hence

$$
\begin{aligned}
& \mathbf{1}^{\top} S_{n, 2}^{(q-1) n} \mathbf{1} \leq \mathbf{1}^{\top} T_{n, 2}^{(q-1)} \mathbf{1} \Rightarrow \\
& \log \rho\left(S_{n, 2}\right)=\lim _{q \rightarrow \infty} \frac{\log \mathbf{1}^{\top} S_{n, 2}^{(q-1) n} \mathbf{1}}{(q-1) n} \leq \\
& \lim _{q \rightarrow \infty} \frac{\mathbf{1}^{\top} T_{n, 2}^{(q-1)} \mathbf{1}}{(q-1) n}=\frac{\log \rho\left(T_{n, 2}\right)}{n} .
\end{aligned}
$$

See [Fri03, Proposition 10.1] for details. This inequality establishes the first part of the upper bound in (I.7). The equality (I.1) yields the inequality

$$
\limsup _{n \rightarrow \infty} \log \rho\left(S_{n, 2}\right) \leq h_{2}(\Delta) .
$$

We now show the inequality $\log \rho\left(S_{n, 2}\right) \leq \frac{\log \rho\left(T_{n+1,2, \text { per }}\right)}{n}$. Let $m=(q-1) n+1$ and consider the colouring of $\langle n\rangle \times\langle q\rangle \cup\{(1, q+1)\}$ induced by colouring $\tau \in \mathrm{C}_{\Delta}(\langle\mathrm{nq}+1\rangle)$ satisfying condition (II.2). Note that the number of such colourings is $\mathbf{1}^{\top} S_{n, 2}^{(q-1) n+1} \mathbf{1}$. We now extend each colouring $\tau$ to the colouring of $\langle n+1\rangle \times\langle q\rangle$ viewed as a colouring of the torus $(\mathbb{Z} /(n+1)) \times\langle q\rangle$ as follows. The colouring of $\langle n\rangle \times\langle q\rangle$ is given by $\tau$ as above. Then the colour of the point $(n+1, i)$ is given by $\tau(i n+1)$ for $i=1, \ldots, q$. Hence $\mathbf{1}^{\top} S_{n, 2}^{(q-1) n+1} \mathbf{1} \leq \mathbf{1}^{\top} T_{n+1,2, \mathrm{per}}^{q-1} \mathbf{1}$. Use [Fri03, Proposition 10.1] as above to deduce the second part of the upper bound in (I.7).

We now show the lower bound in (I.7). Recall that $\mathbf{1}^{\top} T_{n-1,2, \text { per }}^{q-1} \mathbf{1}$ is the number of colourings of the rectangle $\omega:\langle n-1\rangle \times\langle q\rangle$ on the torus $(\mathbb{Z} /(n-1)) \times\langle q\rangle$. Each $\omega$ induces the following $\tau \in \mathrm{C}_{\Delta}(\langle\mathrm{nq}\rangle)$ satisfying (II.2):

$$
\begin{array}{r}
\tau(i+(j-1) n)=\omega((i, j)), \tau(j n)=\omega((1, j)), \\
i=1, \ldots, n-1, j=1, \ldots, q .
\end{array}
$$

Hence $\mathbf{1}^{\top} T_{n-1,2, \operatorname{per}}^{q-1} \mathbf{1} \leq \mathbf{1}^{\top} S_{n, 2}^{(q-1) n} \mathbf{1}$. The above arguments show the lower inequality in (I.7).

Combine the upper bound in (I.3) and the lower bound in (I.7) to obtain

$$
\frac{2 n}{2 n+1} h_{2}(\Delta) \leq \frac{\log \rho\left(T_{2 n, 2, \text { per }}\right)}{2 n+1} \leq \log \rho\left(S_{2 n+1,2}\right) .
$$

Hence $\liminf \operatorname{in}_{n \rightarrow \infty} \log \rho\left(S_{2 n+1,2}\right) \geq h_{2}(\Delta)$. Combine this inequality with (II.3) to deduce (I.6).

\section{The Two DIMENSIONAL RUN LENGTH LIMITED CHANNEL}

We will now apply the above results to the following simple symmetric digraph $\Phi$ on two vertices:

Identify the red colour with the state 1 and the blue colour with the state 2 , which is usually identified with the state 0 . Then $\mathrm{C}_{\Phi}\left(\mathbb{Z}^{\mathrm{d}}\right)$ consists of all colourings of the lattice $\mathbb{Z}^{d}$ in blue and red colours such that no two red colours are adjacent. In terms of codes this correspond to the checkerboard constrain used in e.g. [NZ99] and the simplest hard core model in statistical mechanics.

The adjacency matrix $A(\Phi)$ is $\left[\begin{array}{ll}0 & 1 \\ 1 & 1\end{array}\right]$. It is well known that the one-dimensional entropy corresponding to $\mathrm{C}_{\Phi}(\mathbb{Z})$ is the logarithm of the golden ratio $\frac{1+\sqrt{5}}{2}$. Hence, sometimes $\Phi$ is referred as the golden ratio graph.

\section{A. Computational results}

The topological entropy $h_{2}(\Delta)$ of the hard core model is known within the precision of 10 digits [NZ99]. As in the physics literature, e.g. [Lie67], we will discuss the values of $e^{h_{2}(\Phi)}$. The inequalities obtained in [NZ99] are equivalent to

$$
\begin{aligned}
& 1.50304808247497745859985734 \\
& <e^{h_{2}(\Phi)}< \\
& 1.50304808257186797788004159
\end{aligned}
$$

We have computed eigenvalues for the standard transfer matrix, the periodic transfer matrix and finally for the 1-vertex transfer matrix. Let us examine the results.

Table I gives the computed values of $\rho\left(T_{n, 2}\right), n=2, \ldots, 28$ and $\rho\left(T_{n, 2, \text { per }}\right), n=3, \ldots, 36$. We observed the following facts on the two computed sequences. First the sequence $\frac{\rho\left(T_{n, 2}\right)}{\rho\left(T_{n-1,2}\right)}$ is an increasing sequence for $n \geq 10$. Note that in view of the lower bound given in (I.2) we know that $\frac{\rho\left(T_{2 m, 2}\right)}{\rho\left(T_{2 m-1,2}\right)}$ is a lower bound for $e^{h_{2}(\Phi)}$. Hence

$$
1.50304808247533226432204921 \leq e^{h_{2}(\Phi)} .
$$




\begin{tabular}{|c|c|c|}
\hline$n$ & $\rho\left(T_{n, 2}\right)$ & $\rho\left(T_{n, 2, \text { per }}\right)$ \\
\hline 2 & 2.414213562373095 & \\
\hline 3 & 3.631381260403638 & 3.302775637731994646559610633735247 \\
\hline 4 & 5.457705395965834 & 5.156325174658661693523159039366916 \\
\hline 5 & 8.203259193755024 & 7.637519478750677316156696280583774 \\
\hline 6 & 12.32988221531524 & 11.55170956604814509016646221019832 \\
\hline 7 & 18.53240737754881 & 17.31622927332784947478739705217656 \\
\hline 8 & 27.85509909631079 & 26.05798609193972135567942994470689 \\
\hline 9 & 41.8675533182809 & 39.14578184202813825907509993927013 \\
\hline 10 & 62.928945725187815984970517564242 & 58.85193508152278064182392832406795 \\
\hline 11 & 94.585231204973665631062351227180 & 88.44780432952028084071406736758034 \\
\hline 12 & 142.16615039284113705381555339180 & 132.9477940474849517182393096863462 \\
\hline 13 & 213.68255974084561463042598863826 & 199.8224640440179428924580367714202 \\
\hline 14 & 321.17516167688358891589859286791 & 300.3458520273548324890314287157792 \\
\hline 15 & 482.74171089714185369639722005855 & 451.4321236042340748832899060864991 \\
\hline 16 & 725.58400289480913382756934371728 & 678.5256693463314967782756777455377 \\
\hline 17 & 1090.5876442258184943798276273781 & 1019.855674771119057225300394186046 \\
\hline 18 & 1639.2056674249062545759683245949 & 1532.892835974578082578218246454006 \\
\hline 19 & 2463.8049352057025354985027848902 & 2304.011134761604258032847423262351 \\
\hline 20 & 3703.2172834541914402106141013810 & 3463.039870272410387224398919252810 \\
\hline 21 & 5566.1136368853314325759144845043 & 5205.115189611387576190086821772236 \\
\hline 22 & 8366.1364287602952611690001026376 & 7823.538578192028601159488379441279 \\
\hline 23 & 12574.705316975186002047579953615 & 11759.15453623673826922032891077820 \\
\hline 24 & 18900.386714371918513370098148691 & 17674.57476300121240825274824636313 \\
\hline 25 & 28408.190009078957569653791590282 & 26565.73564566649640784345844846860 \\
\hline 26 & 42698.875519741019043924030420844 & 39929.57806437579207220381471608921 \\
\hline 27 & 64178.462973799644995496644648926 & 60016.07571363818523121568140477488 \\
\hline 28 & 96463.315708983666788807112233379 & 90207.04754031656425802350521860206 \\
\hline 29 & & 135585.5298162229950461965077280634 \\
\hline 30 & & 203791.5706122926277969020818385026 \\
\hline 31 & & 306308.5294259292544744183247215849 \\
\hline 32 & & 460396.4478048022575136612396701088 \\
\hline 33 & & 691997.9980476929053415088280918978 \\
\hline 34 & & 1040106.264045025628338539352181110 \\
\hline 35 & & 1563329.725741562336590052426628794 \\
\hline 36 & & 2349759.746553886953259135919605940 \\
\hline
\end{tabular}

TABLE I

SPECTRAL RADII FOR STANDARD AND PERIODIC TRANSFER MATRICES

The sequence $\rho\left(T_{2 m, 2, \text { per }}\right)^{\frac{1}{2 m}}$ is decreasing for $m=$ $2, \ldots, 18$. In view of the upper bound in (I.3) it follows that

$$
e^{h_{2}(\Phi)} \leq 1.50304808247533992728837255 .
$$

The above two inequalities give the correct 15 digits of the value of $e^{h_{2}(\Phi)}$. Note that the lower bound in (III.1) is closer to the correct value of $e^{h_{2}(\Phi)}$ than the upper bound. It is plausible to assume that the same observation applies to the inequalities (III.2-III.3).

The sequence $\rho\left(T_{2 m+1,2 \text {,per }}\right)^{\frac{1}{2 m+1}}$ increases for $m=$ $1, \ldots, 17$. If this sequence always increases, in view of (I.2), it would follows that $\rho\left(T_{2 m+1,2, \text { per }}\right)^{\frac{1}{2 m+1}}$ is a lower bound for $e^{h_{2}(\Phi)}$. The lower bound given by (III.2) is bigger than $\rho\left(T_{35,2, \text { per }}\right)^{\frac{1}{35}}$.

Table II gives the values of $\rho\left(S_{n, 2}\right)$ for $n=25, \ldots, 40$. We found that the sequence $\rho\left(S_{2 l, 2}\right), l=1, \ldots, 20$ increases while the sequence $\rho\left(S_{2 l-1,2}\right)$ decreases for $l=2, \ldots, 20$. Assuming this behaviour for all values $l \in \mathbb{N}$ we deduce that

$$
\rho\left(S_{2 l, 2}\right)<e^{h_{2}(\Phi)}<\rho\left(S_{2 l-1,2}\right), \text { for all } l \in \mathbb{N} .
$$

The above ansatz for $l=20$ gives the value of $e^{h_{2}(\Phi)}$ with a precision of 15 digits, which coincides with the exact values given by (III.2-III.3). More precisely the heuristic upper bound $\rho\left(S_{39,2}\right)$ is slightly better than (III.3), and the heuristic lower bound given by $\rho\left(S_{40,2}\right)$ is slightly worse than (III.2).

\begin{tabular}{cc}
$n$ & $\rho\left(S_{n, 2}\right)$ \\
\hline Even $n$ & \\
26 & 1.5030480824559338746449982720899 \\
28 & 1.5030480824713491171046098760579 \\
30 & 1.5030480824745080695008293589330 \\
32 & 1.5030480824751605743865692042299 \\
34 & 1.5030480824752962878823092158144 \\
36 & 1.5030480824753246862738777999703 \\
38 & 1.5030480824753306606437859142329 \\
40 & 1.5030480824753319235292607404167 \\
Odd $n$ & \\
39 & 1.5030480824753330032275278142102 \\
37 & 1.5030480824753357484850986619224 \\
35 & 1.5030480824753487657242129983806 \\
33 & 1.5030480824754108025759894900493 \\
31 & 1.5030480824757081424841278582465 \\
29 & 1.5030480824771425174857112752302 \\
27 & 1.5030480824841133358901685021830 \\
25 & 1.5030480825182810708944214989118 \\
& TABLE II
\end{tabular}

SPECTRAL RADII FOR 1-VERTEX TRANSFER MATRICES

\section{B. Computational costs}

We now compare the computational resources needed in order to compute the eigenvalues for the 1-vertex transfer matrix and the standard transfer matrix for cycles. The computations were done in 31 digits precision, both were done on the same machine, and used Perron iteration for computing the maximum eigenvalue. The standard and periodic trans- 
fer matrices were compressed using the method of [LM08] thereby reducing the amount of RAM needed considerably. For the $N=401$-vertex transfer matrix the computation

\begin{tabular}{lll} 
Matrix & Time & RAM \\
\hline$N=40$ 1-vertex & 48 CPU-hours & 0.5GB matrix, two 4GB vectors \\
$N=36$ periodic & 988 CPU-hours & 203GB matrix, two 20MB vectors
\end{tabular}

TABLE III

COMPUTATIONAL COSTS

took 48 CPU-hours, used 0.5GB RAM for the matrix and also needed two vectors or the Perron iteration, each using 4GB RAM. For the $C_{36}$ standard transfer matrix the computation took 988 CPU-hours, the automorphisms compressed transfer matrix used 203GB RAM and also needed two vectors, each using 20MB RAM.

We see that in order to reach a high prescision estimate of the asymptotic capacity the 1-vertex transfer matrix used less memory and less CPU time. The resources needed for the largest 1-vertex transfer matrix used here is today available on many larger workstations, while those needed for the largest periodic transfer matrix still requires a larger cluster. This means that the 1-vertex transfer matrix can first be used on a smaller inexpensive machine in order to find interesting examples of codes so that the more costly large computer will only be used when an interesting candidate has been found.

\section{The General Two Dimensional Case}

Let $\Gamma=(\langle k\rangle, E)$ be a directed graph, abbreviated as digraph, on the set of vertices $V=\langle k\rangle$, where $E \subset\langle k\rangle \times\langle k\rangle$. Let $X \subset \mathbb{Z}$. Then $\phi: X \rightarrow\langle k\rangle$ is viewed as a $k$ colouring of $X . \phi$ is $\Gamma$-allowable colouring of $X$ if $(\phi(i), \phi(i+1)) \in E$ whenever $i, i+1 \in X$. Denote by $\mathrm{C}_{\Gamma}(\mathrm{X})$ the set of allowable $\Gamma$-colourings of $X$. Note that a colour $j \in\langle k\rangle$ appears in $\phi \in \mathrm{C}_{\Gamma}(\mathbb{Z})$ if and only if the vertex $j$ of $\Gamma$ is contained in strongly connected component of $\Gamma$. Hence we assume that $G$ is a disjoint union of strongly connected graphs. Recall that $\log \# \mathrm{C}_{\Gamma}(\langle\mathrm{n}\rangle), \mathrm{n}=1,2, \ldots$, is a subadditive sequence. Hence the $\mathbb{Z}$-entropy induced by $\Gamma$ is given by

$$
h_{1}(\Gamma)=\rho(\Gamma)=\lim _{n \rightarrow \infty} \frac{\log \# \mathrm{C}_{\Gamma}(\langle\mathrm{n}\rangle)}{n},
$$

where $\rho(\Gamma)$ is the spectral radius of $\Gamma$, i.e. the adjacency matrix corresponding to $\Gamma$. See for example [Fri97], [Fri03].

Let $\Gamma_{1}=\left(\langle k\rangle, E_{1}\right), \Gamma_{2}=\left(\langle k\rangle, E_{2}\right), E_{1}, E_{2} \subseteq\langle k\rangle \times\langle k\rangle$ be two directed graphs on the set of vertices $V=\langle k\rangle$. We assume that each $\Gamma_{i}$ is a disjoint union of strongly connected graphs. Let $X \subset \mathbb{Z}^{2}$. For each $i \in \mathbb{Z}$ denote

$$
X_{1, i}=X \cap \mathbb{Z} \times\{i\}, \quad X_{2, i}=\{i\} \times \mathbb{Z} .
$$

View $\phi: X \rightarrow\langle k\rangle$ as a $k$-colouring of $X$. Then $\phi$ is an allowable $\boldsymbol{\Gamma}=\left(\Gamma_{1}, \Gamma_{2}\right)$-colouring of $X$ if $\phi\left|X_{1, i}, \phi\right| X_{2, i}$ are $\Gamma_{1}, \Gamma_{2}$-allowable colourings respectively, for each $i \in \mathbb{Z}$. Let $\mathrm{C}_{\boldsymbol{\Gamma}}(\mathrm{X})$ be all $\boldsymbol{\Gamma}$-allowable colourings of $X$. It is known that the sequence $\log \# \mathrm{C}_{\boldsymbol{\Gamma}}\left(\left\langle\mathrm{n}_{1}\right\rangle \times\left\langle\mathrm{n}_{2}\right\rangle\right)$ is a subadditive sequence in one variable, where the other variable is fixed. Hence the
$\mathbb{Z}^{2}$-entropy induced by $\Gamma$ is given by, e.g. [Fri97], [Fri03],

$$
\begin{aligned}
& h_{2}(\boldsymbol{\Gamma})=\lim _{n_{1}, n_{2} \rightarrow \infty} \frac{\log \# \mathrm{C}_{\boldsymbol{\Gamma}}\left(\left\langle\mathrm{n}_{1}\right\rangle \times\left\langle\mathrm{n}_{2}\right\rangle\right)}{n_{1} n_{2}} \leq \\
& \frac{\log \# \mathrm{C}_{\boldsymbol{\Gamma}}\left(\left\langle\mathrm{n}_{1}\right\rangle \times\left\langle\mathrm{n}_{2}\right\rangle\right)}{n_{1} n_{2}}
\end{aligned}
$$

for all $n_{1}, n_{2} \in \mathbb{N}$.

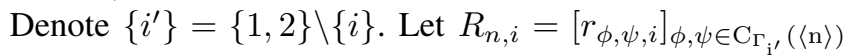
be the following $(0,1)$ transfer matrices for $i=1,2 . r_{\phi, \psi, i}=$ 1 if the colouring of $\langle n\rangle \times\langle 2\rangle$, such that the colouring of $\{(1,1), \ldots,(n, 1)\}$ and $\{(1,2), \ldots,(n, 2)\}$ is given by $\phi$ and $\psi$ respectively, is $\left(\Gamma_{i^{\prime}}, \Gamma_{i}\right)$-allowable. Hence

$$
\begin{aligned}
& \log \rho\left(R_{n, 1}\right)=\lim _{n_{1} \rightarrow \infty} \frac{\log \# \mathrm{C}_{\boldsymbol{\Gamma}}\left(\left\langle\mathrm{n}_{1}\right\rangle \times\langle\mathrm{n}\rangle\right)}{n_{1}}, \\
& \log \rho\left(R_{n, 2}\right)=\lim _{n_{2} \rightarrow \infty} \frac{\log \# \mathrm{C}_{\boldsymbol{\Gamma}}\left(\langle\mathrm{n}\rangle \times\left\langle\mathrm{n}_{2}\right\rangle\right)}{n_{2}}, \\
& h_{2}(\boldsymbol{\Gamma})=\lim _{n \rightarrow \infty} \frac{\log \rho\left(R_{n, i}\right)}{n} \leq \frac{\log \rho\left(R_{n, i}\right)}{n}, \\
& i=1,2, \text { for all } n \in \mathbb{N} .
\end{aligned}
$$

Note that for general $\Gamma_{1}, \Gamma_{2}$ we do not have lower bounds that converge to $h_{2}(\boldsymbol{\Gamma})$. In fact, there are examples that the computation of $h(\boldsymbol{\Gamma})$ is undecidable, e.g. [Fri97], [Fri03]. One has good lower bounds, similar to (I.2), if $\Gamma_{i}$ is undirected for $i=1$ or $i=2$. Note that the isotropic case discussed in $\S 2$ is given by the condition that $\Gamma_{1}=\Gamma_{2}$ is an undirected graph $\Delta$. In that case $T_{n, 2}=R_{n, 1}=R_{n, 2}$.

We now discuss the 1-vertex transfer matrix for a given $\boldsymbol{\Gamma}$. In this case we also have two transfer matrices $P_{n, 1}, P_{n, 2}$ which are $(0,1)$ matrices. For simplicity of the exposition we discuss only $P_{n, 2}$. Let $P_{n, 2}=\left[p_{\phi, \psi, 2}\right]_{\phi, \psi \in \mathrm{C}_{\Gamma_{1}}(\langle\mathrm{n}\rangle)}$. Then $p_{\phi, \psi, 2}=1$ if and only if the conditions given in (I.5) hold, where $E=E_{2}$. The proof of Theorem 2.1 yields the inequality

$$
\log \rho\left(P_{n, 2}\right) \leq \frac{\log \rho\left(R_{n, 2}\right)}{n} \text { for } n \in \mathbb{N} .
$$

Hence

$$
h_{2}(\boldsymbol{\Gamma}) \geq \limsup _{n \rightarrow \infty} \log \rho\left(P_{n, 2}\right) .
$$

It is an interesting question when

$$
h_{2}(\boldsymbol{\Gamma})=\lim _{n \rightarrow \infty} \log \rho\left(P_{n, 2}\right) .
$$

Theorem 2.1 implies that the above equality holds if $\Gamma_{1}=\Gamma_{2}$ is an undirected graph. We now give another condition for the equality (IV.6).

A colour $j \in\langle k\rangle$ is called a friendly colour, if $(j, i),(i, j) \in$ $E_{1} \cap E_{2}$ for all $i \in\langle k\rangle$. The existence of a friendly colour means that $\Gamma_{1}$ and $\Gamma_{2}$ are strongly connected graphs. Note that for the hard core model the colour 2 is friendly.

Theorem 4.1: Let $\Gamma_{1}=\left(\langle k\rangle, E_{1}\right), \Gamma_{2}=\left(\langle k\rangle, E_{2}\right)$ be two digraphs with a friendly colour. Then for each $n \geq 1$

$$
\frac{\log \rho\left(R_{n, 2}\right)}{n+1} \leq \log \rho\left(P_{n+1,2}\right) .
$$

Hence (IV.6) holds.

Proof: As in the proof of Theorem 2.1 we deduce that

$$
\# \mathrm{C}_{\boldsymbol{\Gamma}}(\langle\mathrm{n}\rangle \times\langle\mathrm{m}\rangle)=\mathbf{1}^{\top} \mathrm{R}_{\mathrm{n}, 2}^{\mathrm{m}-1} \mathbf{1} .
$$


Observe next that $\mathbf{1}^{\top} P_{n+1,2}^{(n+1)(m-1)} \mathbf{1}$ is the number of all $\omega \in$ $\mathrm{C}_{\Gamma_{1}}(\langle(\mathrm{n}+1) \mathrm{m}\rangle)$ such that $(\omega(i), \omega(n+1+i)) \in E_{2}$ for $i=1, \ldots,(m-1)(n+1)$. Let $\tau \in \mathrm{C}_{\boldsymbol{\Gamma}}(\langle\mathrm{n}\rangle \times\langle\mathrm{m}\rangle)$. Extend $\tau$ to $\hat{\tau}:\langle n+1\rangle \times m$ by letting $\hat{\tau}((n+1, i))=j$ for $i=$ $1, \ldots, m$, where $j$ is a friendly colour of $\boldsymbol{\Gamma}$. Then each such $\hat{\tau}$ is induced by $\omega \in \mathrm{C}_{\Gamma_{1}}(\langle(\mathrm{n}+1) \mathrm{m}\rangle)$ satisfying the above additional conditions. Hence

$$
\mathbf{1}^{\top} R_{n, 2}^{m-1} \mathbf{1} \leq \mathbf{1}^{\top} P_{n+1,2}^{(n+1)(m-1)} \mathbf{1} .
$$

The arguments of the proof of Theorem 2.1 yield the inequality (IV.7). Combine this inequality with the inequality (IV.5) and the characterisation of $h_{2}(\boldsymbol{\Gamma})$ in (IV.3) to deduce (IV.6).

\section{THE THREE DIMENSIONAL CASE}

Let $\Gamma_{i}=\left(\langle k\rangle, E_{i}\right), E_{i} \subseteq\langle k\rangle \times\langle k\rangle$ be a digraph, which is a disjoint union of strongly connected graphs, for $i=1,2,3$. Denote $\boldsymbol{\Gamma}=\left(\Gamma_{1}, \Gamma_{2}, \Gamma_{3}\right)$. Let $\mathbf{e}_{i}=\left(\delta_{1 i}, \delta_{i 2}, \delta_{i 3}\right)^{\top}, i=1,2,3$ be the standard basis in $\mathbb{Z}^{3}$. For $X \subset \mathbb{Z}^{3}$ the colouring $\phi: X \rightarrow\langle k\rangle$ is called $\boldsymbol{\Gamma}$-allowable if $\left(\phi(\mathbf{j}), \phi\left(\mathbf{j}+\mathbf{e}_{i}\right)\right) \in E_{i}$, whenever $\mathbf{j}, \mathbf{j}+\mathbf{e}_{i} \in X$, for $\mathbf{j} \in \mathbb{Z}^{3}$ and $i=1,2,3$. Denote by $\mathrm{C}_{\boldsymbol{\Gamma}}(\mathrm{X})$ all $\Gamma$-allowable colourings of $X$. Recall that the sequence $\log \# \mathrm{C}_{\Gamma}\left(\left\langle\mathrm{n}_{1}\right\rangle \times\left\langle\mathrm{n}_{2}\right\rangle \times\left\langle\mathrm{n}_{3}\right\rangle\right)$ is a subadditive sequence in one variable, where the other variables are fixed. Hence the $\mathbb{Z}^{3}$-entropy induced by $\Gamma$ is given by, e.g. [Fri97], [Fri03],

$$
\begin{aligned}
h_{3}(\boldsymbol{\Gamma})=\lim _{n_{1}, n_{2}, n_{3} \rightarrow \infty} & \frac{\log \# \mathrm{C}_{\boldsymbol{\Gamma}}\left(\left\langle\mathrm{n}_{1}\right\rangle \times\left\langle\mathrm{n}_{2}\right\rangle \times\left\langle\mathrm{n}_{3}\right\rangle\right)}{n_{1} n_{2} n_{3}} \\
\leq & \frac{\log \# \mathrm{C}_{\boldsymbol{\Gamma}}\left(\left\langle\mathrm{n}_{1}\right\rangle \times\left\langle\mathrm{n}_{2}\right\rangle \times\left\langle\mathrm{n}_{3}\right\rangle\right)}{n_{1} n_{2} n_{3}},
\end{aligned}
$$

for all $n_{1}, n_{2}, n_{3} \in \mathbb{N}$. Let $\mathbf{n}=\left(n_{1}, n_{2}\right) \in \mathbb{N}^{2}$. Denote $\langle\mathbf{n}\rangle=$ $\left\langle n_{1}\right\rangle \times\left\langle n_{2}\right\rangle$. As in the two dimensional case one can define the transfer matrices $R_{\mathbf{n}, 1}, R_{\mathbf{n}, 2}, R_{\mathbf{n}, 3}$ which are $(0,1)$ matrices. For simplicity of the exposition we discuss only

$$
R_{\mathbf{n}, 3}=\left[r_{\phi, \psi, 3}\right]_{\phi, \psi \in \mathrm{C}_{\left(\Gamma_{1}, \Gamma_{2}\right)}(\langle\mathbf{n}\rangle)} .
$$

$r_{\phi, \psi, 3}=1$ if the colouring of $\langle\mathbf{n}\rangle \times\langle 2\rangle$, such that the colouring of $\langle\mathbf{n}\rangle \times\{1\},\langle\mathbf{n}\rangle \times\{2\}$ given by $\phi$ and $\psi$ respectively, is $\boldsymbol{\Gamma}$ allowable. Hence

$$
\begin{aligned}
& \log \rho\left(R_{\left(n_{1}, n_{2}\right), 3}\right)=\lim _{n_{3} \rightarrow \infty} \frac{\log \# \mathrm{C}_{\boldsymbol{\Gamma}}\left(\left\langle\mathrm{n}_{1}\right\rangle \times\left\langle\mathrm{n}_{2}\right\rangle \times \mathrm{n}_{3}\right)}{n_{3}}, \\
& \left.h_{3}(\boldsymbol{\Gamma})=\lim _{n_{1}, n_{2} \rightarrow \infty} \frac{\log \rho\left(R_{\left(n_{1}, n_{2}\right), 3}\right)}{n_{1} n_{2}} \leq \frac{\left.\log \rho\left(R_{\left(n_{1}, n_{2}\right.}()_{3}\right) .2\right)}{n_{1} n_{2}}\right)
\end{aligned}
$$

for all $n_{1}, n_{2} \in \mathbb{N}$.

We now discuss the 1-vertex transfer matrix for a given $\Gamma$. In this case we have three transfer matrices $P_{\mathbf{n}, 1}, P_{\mathbf{n}, 2}, P_{\mathbf{n}, 3}$ $(0,1)$ matrices. For simplicity of the exposition we discuss only $P_{\mathbf{n}, 3}$. Let $\hat{\mathrm{C}}_{\Gamma_{1}}\left(\left\langle n_{1} n_{2}\right\rangle\right)$ be the subset of all $\Gamma_{1}$-allowable colourings of $\phi \in \mathrm{C}_{\Gamma_{1}}\left(\left\langle\mathrm{n}_{1} \mathrm{n}_{2}\right\rangle\right)$ satisfying the additional condition:

$$
\left(\phi(i), \phi\left(n_{1}+i\right)\right) \in E_{2} \text { for } i=1, \ldots, n_{1}\left(n_{2}-1\right) .
$$

The condition (V.3) yields that the colouring given by $\phi$ on $\left\langle n_{1} n_{2}\right\rangle$ induces a $\left(\Gamma_{1}, \Gamma_{2}\right)$-allowable colouring of $\langle\mathbf{n}\rangle \times\{1\}$ as discussed in the previous section.
Let $P_{\mathbf{n}, 3}=\left[p_{\phi, \psi, 3}\right]_{\phi, \psi \in \hat{\mathrm{C}}_{\Gamma_{1}}\left(\left\langle n_{1} n_{2}\right\rangle\right)}$. Then $p_{\phi, \psi, 3}=1$ if and only if $\psi(i)=\phi(i+1)$ for $i=1, \ldots, n_{1} n_{2}-1$ and

$$
\left(\phi(1), \psi\left(n_{1} n_{2}\right)\right) \in E_{3} .
$$

The condition (V.4) yields that if we colour $\langle\mathbf{n}\rangle \times 1$ in $\phi$ and $(1,1,2)$ in the colour $\psi\left(n_{1} n_{2}\right)$, we obtain a $\boldsymbol{\Gamma}$-allowable colouring of $\langle\mathbf{n}\rangle \times\{1\} \cup\{(1,1,2)\}$.

Observe that $\mathbf{1}^{\top} P_{\mathbf{n}, 3}^{n_{1} n_{2}(m-1)} \mathbf{1}$ consists of all $\boldsymbol{\omega} \in$ $\mathrm{C}_{\Gamma_{1}}\left(\mathrm{n}_{1} \mathrm{n}_{2} \mathrm{~m}\right)$ satisfying the above "periodic" conditions

1) $\left(\omega(i), \omega\left(i+n_{1}\right)\right) \in E_{2}, i=1, \ldots, n_{1}\left(n_{2} m-1\right)$.

2) $\left(\omega(i), \omega\left(i+n_{1} n_{2}\right) \in E_{3}, i=1, \ldots, n_{1} n_{2}(m-1)\right.$.

Hence each such $\omega$ induces a $\boldsymbol{\Gamma}$-allowable colouring of $\langle\mathbf{n}\rangle \times$ $\langle m\rangle$. Therefore

$$
\mathbf{1}^{\top} P_{\mathbf{n}, 3}^{n_{1} n_{2}(m-1)} \mathbf{1} \leq \mathbf{1}^{\top} R_{\mathbf{n}, 3}^{m-1} \mathbf{1} .
$$

The proof of Theorem 2.1 yields the inequality

$$
\log \rho\left(P_{\left(n_{1}, n_{2}\right), 3}\right) \leq \frac{\log \rho\left(R_{\left(n_{1}, n_{2}\right), 3}\right)}{n_{1} n_{2}} \text { for } n_{1}, n_{2} \in \mathbb{N} \text {. }
$$

Hence

$$
h_{3}(\boldsymbol{\Gamma}) \geq \limsup _{n_{1}, n_{2} \rightarrow \infty} \log \rho\left(P_{\mathbf{n}, 3}\right) .
$$

It is an interesting question when

$$
h_{3}(\boldsymbol{\Gamma})=\lim _{n_{1}, n_{2} \rightarrow \infty} \log \rho\left(P_{\left(n_{1}, n_{2}\right), 3}\right) .
$$

A colour $j \in\langle k\rangle$ is called a friendly colour, if $(j, i),(i, j) \in$ $E_{1} \cap E_{2} \cap E_{3}$ for all $i \in\langle k\rangle$.

Theorem 5.1: Let $\Gamma_{1}=\left(\langle k\rangle, E_{1}\right), \Gamma_{2}=\left(\langle k\rangle, E_{2}\right), \Gamma_{3}$ be three digraphs with a friendly colour. Then for each $n_{1}, n_{2} \geq 1$

$$
\frac{\log \rho\left(R_{\left(n_{1}, n_{2}\right), 3}\right)}{\left(n_{1}+1\right)\left(n_{2}+1\right)} \leq \log \rho\left(P_{\left(n+1, n_{2}+1\right), 3}\right) .
$$

Hence (V.6) holds.

Proof: Let $\phi \in \mathrm{C}_{\boldsymbol{\Gamma}}\left(\left\langle\mathrm{n}_{1}\right\rangle \times\left\langle\mathrm{n}_{2}\right\rangle \times\langle\mathrm{m}\rangle\right)$. Extend $\phi$ to the colouring $\hat{\phi}:\left\langle n_{1}+1\right\rangle \times\left\langle n_{2}+1\right\rangle \times\langle m\rangle$ by colouring the additional points in a friendly colour. Note that $\hat{\phi}$ induces the colouring $\omega \in \mathrm{C}_{\Gamma_{1}}\left(\left(\mathrm{n}_{1}+1\right)\left(\mathrm{n}_{2}+1\right) \mathrm{m}\right)$ such that

$$
\begin{array}{r}
\left(\omega(i), \omega\left(i+n_{1}+1\right)\right) \in E_{2}, \\
i=1, \ldots,\left(n_{1}+1\right)\left(n_{2}+1\right) m-n_{1}-1, \\
\left(\omega(j), \omega\left(j+\left(n_{1}+1\right)\left(n_{2}+1\right)\right)\right) \in E_{3}, \\
j=1, \ldots\left(n_{1}+1\right)\left(n_{2}+1\right)(m-1) .
\end{array}
$$

Hence

$$
\mathbf{1}^{\top} R_{\left(n_{1}, n_{2}\right), 3}^{m-1} \mathbf{1} \mathbf{1}^{\top} P_{\left(n_{1}+1, n_{2}+1\right), 3}^{\left(n_{1}+1\right)\left(n_{2}+1\right)(m-1)} \mathbf{1} .
$$

Therefore (V.7) holds. Use (V.5) and (V.2) to deduce (V.6).

We now discuss briefly the isotropic symmetric case, i.e. $\Gamma_{1}=\Gamma_{2}=\Gamma_{3}=\Delta=(\langle k\rangle, E)$ is an undirected graph with no isolated vertices. Then the transfer matrix $T_{\left(n_{1}, n_{2}\right), 3}$ is equal to $R_{\left(n_{1}, n_{2}\right), 3}$. Denote by $T_{\left(n_{1}, n_{2}\right), 3 \text {,per }}$ the transfer matrix corresponding to all $\left(n_{1}, n_{2}\right)$ periodic colourings, which are $\Delta$-allowable colourings of $\left(\mathbb{Z} / n_{1}\right) \times\left(\mathbb{Z} / n_{2}\right)$. Note that 
$T_{\left(n_{1}, n_{2}\right), 3, \text { per }}$ is a principal submatrix of $T_{\left(n_{1}, n_{2}\right), 3}$. Recall the following improvement of the (V.2) [Fri97], [Fri03], [FP05]

$$
\begin{aligned}
h_{3}(\boldsymbol{\Gamma})=\lim _{n_{1}, n_{2} \rightarrow \infty} \frac{\log \rho\left(T_{\left(2 n_{1}, 2 n_{2}\right), 3, \mathrm{per}}\right)}{4 n_{1} n_{2}} \leq \\
\frac{\log \rho\left(T_{\left(2 n_{1}, 2 n_{2}\right), 3, \mathrm{per}}\right)}{4 n_{1} n_{2}},
\end{aligned}
$$

for all $n_{1}, n_{2} \in \mathbb{N}$.

Let $S_{\left(n_{1}, n_{2}\right), 3}=P_{\left(n_{1}, n_{2}\right), 3}$ be the 1-vertex transfer matrix corresponding to $\Delta$. A given $\Delta$-allowable colouring on $\mathbb{Z} \times$ $\mathbb{Z} \times\langle m\rangle$, which is $\left(n_{1}, n_{2}\right)$ periodic on each $\mathbb{Z} \times \mathbb{Z} \times\{i\}$ for each $i \in\langle m\rangle$, induces a colouring $\phi \in \mathrm{C}_{\Delta}\left(\left\langle\mathrm{n}_{1}+1\right\rangle \times\right.$ $\left.\left\langle\mathrm{n}_{2}+1\right\rangle \times\langle\mathrm{m}\rangle\right)$. This colouring corresponds to the colouring $\omega \in \mathrm{C}_{\Delta}\left(\left(\mathrm{n}_{1}+1\right)\left(\mathrm{n}_{2}+1\right) \mathrm{m}\right)$ that satisfies the conditions (V.9), where $E_{2}=E_{3}=E$. Hence we have an analogue of (V.7)

$$
\frac{\log \rho\left(T_{\left(n_{1}, n_{2}\right), 3, \text { per }}\right)}{\left(n_{1}+1\right)\left(n_{2}+1\right)} \leq \log \rho\left(S_{\left(n_{1}+1, n_{2}+1\right), 3}\right) .
$$

Use (V.11), (V.5) and (V.2) to deduce

$$
\lim _{n_{1}, n_{2} \rightarrow \infty} \log \rho\left(S_{\left(2 n_{1}+1,2 n_{2}+1\right), 3}\right)=h_{3}(\Delta) .
$$

\section{The Three-Dimensional Run Length Limited CHANNEL}

The capacity of the three-dimensional channel corresponding to the isotropic symmetric case of $\mathbb{Z}^{3}$ with the same digraph as in Section III is the three-dimensional $(0,1)$ run length limited channel. This channel was considered in [NZ03] where the transfer matrix methods previously used for the twodimensional case was extended to find the bounds

$$
1.43644 \leq e^{h_{3}(\boldsymbol{\Gamma})} \leq 1.44082
$$

This case has also been studied in the physics literature, see e.g. [MLA07], in the form of a three-dimensional lattice gas, where colourings are weighted according to their number of 1s. Mathematical results one the structure of the set of colourings have shown [Gal08], [GK04] that many of the ordinary Monte Carlo algorithms used to study this model numerically have slow mixing properties and that there are interesting long range correlations in the positions of the $1 \mathrm{~s}$.

\section{A. Computational results}

We first used the standard transfer matrix method in combination with the compression method of [LM08] to compute the largest eigenvalue for both the periodic and aperiodic graphs needed to bound the capacity for this channel. In order to verify our program we recomputed the eigenvalues from [NZ03] and the results agreed. In Table IV we show the eigenvalues not previously computed. Using these eigenvalues as in [NZ99], [FP05] we find the following bounds for the exponentiated capacity:

$$
1.4365871627266 \leq e^{h_{3}(\boldsymbol{\Gamma})} \leq 1.43781634614
$$

We have here determined one more decimal in the value of $e^{h_{3}(\boldsymbol{\Gamma})}$, and limited the next decimal to only two possible values.
The largest eigenvalues for the 1-vertex transfer are given in Table V. As we can see the eigenvalues give a reasonably good approximation of the capacity. Even for a small case like $(4,4)$ the first three decimals agree with the bounds in VI.1. For the largest case the eigenvalue is in the interval given in VI.1, but here we do not have an error bound. At first sight the values given by the 1-vertex transfer matrix looks less impressive for this case, but in fact the best bounds before the current paper only gave $e^{h_{3}(\boldsymbol{\Gamma})}=1$.4. Hence even the $(4,4)$ case for the 1-vertex transfer matrix gave an estimate with improved accuracy.

\begin{tabular}{lll}
$n_{1}$ & $n_{2}$ & \\
\hline 4 & 4 & 1.431707 \\
4 & 5 & 1.433880 \\
5 & 4 & 1.433943 \\
5 & 5 & 1.439764 \\
6 & 5 & 1.436801
\end{tabular}

TABLE V

LARGEST EIGENVALUE FOR THE 1-VERTEX TRANSFER MATRIX

\section{B. Computational costs}

The most striking feature for the three-dimensional case is the difference in computational resources needed for the two approaches.

The computation of the eigenvalues for $C_{6,8}$ used 3700 GB RAM and ran for 10.6 hours on 512 4-core CPUs on a linux cluster, giving a total of about 21700 CPU-hours. The program was a Fortan 90 program using OpenMP and MPI for communication. Before compression the matrix had side 1682382.

For the 1-vertex transfer matrix constraints, in programming time and computer access, made us settle for a single machine implementation of the method in Mathematica which was run on a desktop Macintosh with 2GB RAM. For $n_{1}=6, n_{2}=5$ the computation used 52 CPU-hours. The matrix has side 339 000 but since it has only two non-zero positions in each row the amount of RAM used was negligible.

\begin{tabular}{lll} 
Matrix & Time & RAM \\
\hline$n_{1}=6, n_{2}=51$-vertex & 52 CPU-hours & 5MB matrix \\
$C_{6} \times C_{8}$ periodic & 21700 CPU-hours & 3700GB matrix \\
\multicolumn{3}{c}{ COMPUTATIONAL COSTS FOR THREE-DIMENSIONS }
\end{tabular}

\section{THE MONOMER-DIMER MODEL}

The classical monomer-dimer model on $\mathbb{Z}^{d}$ consists of tiling $\mathbb{Z}^{d}$ with monomers and dimers in the direction $\mathbf{e}_{i}=$ $\left(\delta_{1 i}, \ldots, \delta_{d i}\right)^{\top}$ for $i=1, \ldots, d$. This tiling can be coded in $2 d+1$ colours, see [Fri03], [FP05]. That is, the colour $2 d+1$ corresponds to a monomer, and the colours $2 i-1,2 i$ correspond a dimer in the direction $\mathbf{e}_{i}$. So $\Gamma_{i}=\left(\langle 2 d+1\rangle, E_{i}\right)$ is given by the following conditions on $E_{i}$ for $i=1, \ldots, d$.

- All vertices $\langle 2 d+1\rangle \backslash\{2 i-1,2 i\}$ form a complete directed graph on $2 d-1$ vertices.

- $(j, 2 i-1),(2 i, j) \in E_{i}$ for all $j \in\langle 2 d+1\rangle \backslash\{2 i-1,2 i\}$. 


\begin{tabular}{lllll}
$n_{1}$ & $n_{2}$ & aperiodic & periodic & aperiodic/periodic \\
\hline 3 & 12 & & & 769999.6006127802 \\
\hline 4 & 7 & 41543.31662520356 & & \\
4 & 8 & 184654.5439467464 & 117151.9963311473 & \\
4 & 10 & & 215347.9226316121 & \\
\hline 5 & 5 & 13427.06985344107 & 8185.111027254276 & 10331.06553679985 \\
5 & 6 & 85738.84889761954 & 51133.96879131764 & 68793.17993810770 \\
5 & 7 & 547489.0319884565 & 307078.2654460451 & \\
5 & 8 & & & 2779905.231816480 \\
\hline 6 & 6 & 786528.5060953929 & 482862.3074476483 & \\
6 & 8 & & 37133338.84386827 &
\end{tabular}

TABLE IV

LARGEST EIGENVALUE FOR THE STANDARD TRANSFER MATRIX

- $(2 i-1,2 i) \in E_{i}$.

Denote by $h_{d}$ the monomer-dimer entropy, e.g. [Fri03], [FP05]. Let $\mathbf{n}=\left(n_{1}, \ldots, n_{d-1}\right) \in \mathbb{N}^{d-1}$. Define the 1 -vertex transfer matrix $P_{\mathbf{n}, d}$ as in the previous sections for $d=2,3$. We claim that

$$
h_{d}=\lim _{n_{1}, \ldots, n_{d-1} \rightarrow \infty} \log \rho\left(P_{\left(n_{1}, \ldots, n_{d-1}\right), d}\right) .
$$

In this case the colour $2 d+1$ is not friendly. It is shown in [FP05] that to compute the $d$-dimer entropy $h_{d}$ it is enough to consider the tiling of the box $\phi$ of $\left\langle n_{1}\right\rangle \times \ldots \times\left\langle n_{d}\right\rangle$ with monomers and dimers that fit inside the box. In this case we can extend the tiling $\phi$ to the tiling $\tilde{\phi}$ of $\left\langle n_{1}+1\right\rangle \times \ldots \times\left\langle n_{d-1}+\right.$ $1\rangle \times\left\langle n_{d}\right\rangle$ by adding an additional layer of monomers. The arguments of the previous section yield the equality (VII.1).

\section{ACKNOWLEDGEMENTS}

This research was conducted using the resources of High Performance Computing Center North (HPC2N).

\section{REFERENCES}

$\left[\mathrm{BCL}^{+} 06\right]$ Christian Borgs, Jennifer Chayes, László Lovász, Vera T. Sós, and Katalin Vesztergombi, Counting graph homomorphisms, Topics in discrete mathematics, Algorithms Combin., vol. 26, Springer, Berlin, 2006, pp. 315-371. MR MR2249277 (2007f:05087)

[BET80] R. J. Baxter, I. G. Enting, and S. K. Tsang, Hard-square lattice gas, J. Statist. Phys. 22 (1980), no. 4, 465-489. MR MR574008 (81f:82037)

[CW98] Neil J. Calkin and Herbert S. Wilf, The number of independent sets in a grid graph, SIAM J. Discrete Math. 11 (1998), no. 1, 54-60 (electronic). MR MR1612853 (99e:05010)

[FLS07] Michael Freedman, László Lovász, and Alexander Schrijver, Reflection positivity, rank connectivity, and homomorphism of graphs, J. Amer. Math. Soc. 20 (2007), no. 1, 37-51 (electronic). MR MR2257396 (2007h:05153)

[FP05] Shmuel Friedland and Uri N. Peled, Theory of computation of multidimensional entropy with an application to the monomerdimer problem, Adv. in Appl. Math. 34 (2005), no. 3, 486-522. MR MR2123547 (2005m:82020)

[Fri97] Shmuel Friedland, On the entropy of $\mathbf{Z}^{d}$ subshifts of finite type, Linear Algebra Appl. 252 (1997), 199-220. MR MR1428636 (98b:54052)

[Fri03] - Multi-dimensional capacity, pressure and Hausdorff dimension, Mathematical systems theory in biology, communications, computation, and finance (Notre Dame, IN, 2002), IMA Vol. Math. Appl., vol. 134, Springer, New York, 2003, pp. 183222. MR MR2043239 (2004m:37027)

[Ga108] David Galvin, Sampling independent sets in the discrete torus, Random Structures Algorithms 33 (2008), no. 3, 356-376. MR MR2446486

[GK04] David Galvin and Jeff Kahn, On phase transition in the hardcore model on $\mathbf{Z}^{d}$, Combin. Probab. Comput. 13 (2004), no. 2, 137-164. MR MR2047233 (2005c:82034)
[KZ99] Akiko Kato and Kenneth Zeger, On the capacity of twodimensional run-length constrained channels, IEEE Trans. Inform. Theory 45 (1999), no. 5, 1527-1540. MR MR1699075 (2000c:94012)

[Lie67] E. H. Lieb, Residual Entropy of Square Ice, Physical Review 162 (1967), 162-172.

[LM08] Per Håkan Lundow and Klas Markström, Exact and approximate compression of transfer matrices for graph homomorphisms, LMS J. Comput. Math. 11 (2008), 1-14. MR MR2379936 (2009e:05192)

[MLA07] H. C. Marques Fernandes, Y. Levin, and J. J. Arenzon, Equation of state for hard-square lattice gases, Physical Review E 75 (2007), no. 5, 052101-+.

[NZ99] Zsigmond Nagy and Kenneth Zeger, Capacity bounds for the 3dimensional $(0,1)$ runlength limited channel, Applied algebra, algebraic algorithms and error-correcting codes (Honolulu, HI, 1999), Lecture Notes in Comput. Sci., vol. 1719, Springer, Berlin, 1999, pp. 245-251. MR MR1846500 (2002i:94023)

[NZ03] Asymptotic capacity of two-dimensional channels with checkerboard constraints, IEEE Trans. Inform. Theory 49 (2003), no. 9, 2115-2125. MR MR2004767 (2005g:94061) 